\title{
Category theoretic semantics for theorem proving in logic programming: embracing the laxness
}

\author{
Ekaterina Komendantskaya ${ }^{1}$ and John Power $^{2}$ \\ 1 Department of Computing, University of Dundee, UK ${ }^{\star}$ \\ 2 Department of Computer Science, University of Bath, UK **
}

\begin{abstract}
A propositional logic program $P$ may be identified with a $P_{f} P_{f}$-coalgebra on the set of atomic propositions in the program. The corresponding $C\left(P_{f} P_{f}\right)$-coalgebra, where $C\left(P_{f} P_{f}\right)$ is the cofree comonad on $P_{f} P_{f}$, describes derivations by resolution. Using lax semantics, that correspondence may be extended to a class of first-order logic programs without existential variables. The resulting extension captures the proofs by term-matching resolution in logic programming. Refining the lax approach, we further extend it to arbitrary logic programs. We also exhibit a refinement of Bonchi and Zanasi's saturation semantics for logic programming that complements lax semantics.

Keywords: Logic programming, coalgebra, term-matching resolution, coinductive derivation tree, Lawvere theories, lax transformations, Kan extensions.
\end{abstract}

\section{Introduction}

Consider the following two logic programs.

Example 1. ListNat (for lists of natural numbers) denotes the logic program

1. nat $(0) \leftarrow$

2. $\operatorname{nat}(\mathrm{s}(\mathrm{x})) \leftarrow \operatorname{nat}(\mathrm{x})$

3. list(nil) $\leftarrow$

4. list $(\operatorname{cons}(\mathrm{x}, \mathrm{y})) \leftarrow \operatorname{nat}(\mathrm{x}), \operatorname{list}(\mathrm{y})$

Example 2. GC (for graph connectivity) denotes the logic program

0 . connected $(\mathrm{x}, \mathrm{x}) \leftarrow$

1. connected $(x, y) \leftarrow$ edge $(x, z)$, connected $(z, y)$

* Ekaterina Komendantskaya would like to acknowledge the support of EPSRC Grant $\mathrm{EP} / \mathrm{K} 031864 / 1$.

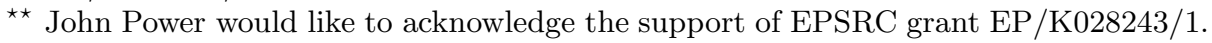
No data was generated in the course of this research. 
A critical difference between ListNat and GC is that in the latter, which is a leading example in Sterling and Shapiro's book [31, there is a variable $z$ in the tail of the second clause that does not appear in its head. The category theoretic consequences of that fact are the central concern of this paper.

It has long been observed, e.g., in [48], that logic programs induce coalgebras, allowing coalgebraic modelling of their operational semantics. In 20, we developed the idea for variable-free logic programs as follows. Using the definition of a logic program [25, given a set of atoms $A t$, one can identify a variable-free logic program $P$ built over $A t$ with a $P_{f} P_{f}$-coalgebra structure on $A t$, where $P_{f}$ is the finite powerset functor on $S e t$ : each atom is the head of finitely many clauses in $P$, and the body of each clause contains finitely many atoms. Our main result was that if $C\left(P_{f} P_{f}\right)$ is the cofree comonad on $P_{f} P_{f}$, then, given a logic program $P$ qua $P_{f} P_{f}$-coalgebra, the corresponding $C\left(P_{f} P_{f}\right)$-coalgebra structure characterises the and-or derivation trees generated by $P, \operatorname{cf}[12$.

This result has proved to be stable, not only having been further developed by us 232415|9|10, but also forming the basis for Bonchi and Zanasi's saturation semantics for logic programming (LP) 617. In Sections 2, 3, we give an updated account of the work, with updated definitions, proofs and detailed examples, to start our semantic analysis of derivations and proofs in LP.

In [21, we extended our analysis from variable-free logic programs to arbitrary logic programs. Following [1/45]19, given a signature $\Sigma$ of function symbols, we let $\mathcal{L}_{\Sigma}$ denote the Lawvere theory generated by $\Sigma$, and, given a logic program $P$ with function symbols in $\Sigma$, we considered the functor category $\left[\mathcal{L}_{\Sigma}^{o p}, S e t\right]$, extending the set $A t$ of atoms in a variable-free logic program to the functor from $\mathcal{L}_{\Sigma}^{o p}$ to Set sending a natural number $n$ to the set $A t(n)$ of atomic formulae with at most $n$ variables generated by the function symbols in $\Sigma$ and the predicate symbols in $P$. We sought to model $P$ by a $\left[\mathcal{L}_{\Sigma}^{o p}, P_{f} P_{f}\right]$-coalgebra $p: A t \longrightarrow P_{f} P_{f} A t$ that, at $n$, takes an atomic formula $A\left(x_{1}, \ldots, x_{n}\right)$ with at most $n$ variables, considers all substitutions of clauses in $P$ into clauses with variables among $x_{1}, \ldots, x_{n}$ whose head agrees with $A\left(x_{1}, \ldots, x_{n}\right)$, and gives the set of sets of atomic formulae in antecedents, mimicking the construction for variable-free logic programs. Unfortunately, that idea was too simple.

Consider the logic program ListNat, i.e., Example1, There is a map in $\mathcal{L}_{\Sigma}$ of the form $0 \rightarrow 1$ that models the nullary function symbol 0 . So, naturality of the map $p: A t \longrightarrow P_{f} P_{f} A t$ in $\left[\mathcal{L}_{\Sigma}^{o p}, S e t\right]$ would yield commutativity of the diagram

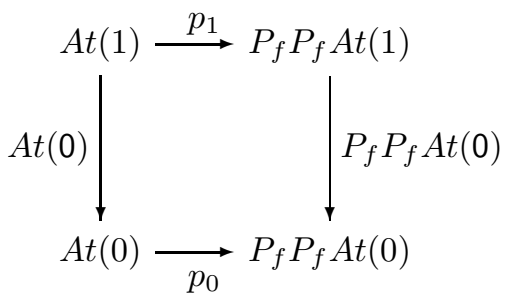

But consider nat $(\mathrm{x}) \in A t(1)$ : there is no clause of the form nat $(\mathrm{x}) \leftarrow$ in ListNat, so commutativity of the diagram would imply that there cannot be a clause in ListNat of the form nat $(0) \leftarrow$ either, but in fact there is one. 
At that point, proposed resolutions diverged: at CALCO in 2011, we proposed one approach using lax transformations [21, then at CALCO 2013, Bonchi and Zanasi proposed another, using saturation semantics [6], an example of the positive interaction generated by CALCO! In fact, as we explain in Section 6, the two approaches may be seen as complementary rather than as alternatives. First we shall describe our approach.

We followed the standard category theoretic technique of relaxing the naturality condition on $p$ to a subset condition, e.g., as in [2]3|13|16]18, so that, in general, given a map in $\mathcal{L}_{\Sigma}$ of the form $f: n \rightarrow m$, the diagram

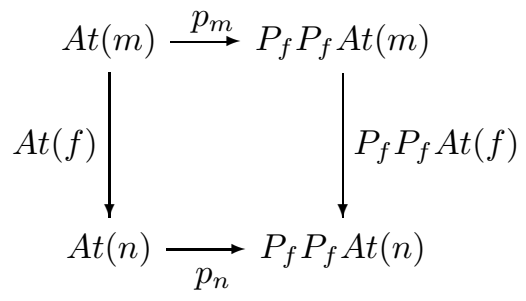

need not commute, but rather the composite via $P_{f} P_{f} A t(m)$ need only yield a subset of that via $A t(n)$. So, for example, $p_{1}(\operatorname{nat}(\mathrm{x}))$ could be the empty set while $p_{0}($ nat $(0))$ could be non-empty in the semantics for ListNat as required. We extended Set to Poset in order to express the laxness, and we adopted established category theoretic research on laxness, notably that of [16, in order to prove that a cofree comonad exists and behaves as we wished.

For a representative class of logic programs, the above semantics describes derivations arising from restricting the usual SLD-resolution used in LP to termmatching resolution, cf. [22 23]. As transpired in further studies [915, this particular restriction to resolution rule captures the theorem-proving aspect of LP as opposed to the problem-solving aspect captured by SLD-resolution with unification. We explain this idea in Section 2, Derivation trees arising from proofs by term-matching resolution were called coiductive trees in 22123] to mark their connection to the coalgebraic semantics.

Categorical semantics introduced in 21] worked well for ListNat, allowing us to model its coinductive trees, as we show in Section 4 (It was not shown explicitly in [21]). However, it does not work well for GC, the key difference being that, in ListNat, no variable appears in a tail of a clause that does not also appear in its head, i.e., clauses in ListNat contain no existential variables. In contrast, although not expressed in these terms in 21, we were unable to model the coinductive trees generated by GC because it is an existential program, i.e. program containing clauses with existential variables. We worked around the problems in 21, but only inelegantly.

We give an updated account of 21] in Section 4, going beyond 21] to explain how coinductive trees for logic programs without existential variables are modelled, and explaining the difficulty in modelling coinductive semantics for arbitrary logic programs. We then devote Section 5 of the paper to resolution of the difficulty, providing lax semantics for coinductive trees generated by arbitrary logic progams. 
In contrast to this, Bonchi and Zanasi, concerned by the complexity involved with laxness, proposed the use of saturation, following [4, to provide an alternative category theoretic semantics [6]7. Saturation is indeed an established and useful construct, as Bonchi and Zanasi emphasised [67,, with a venerable tradition, and, as they say, laxness requires careful calculation, albeit much less so in the setting of posets than that of categories. On the other hand, laxness is a standard part of category theory, one that has been accepted by computer scientists as the need has arisen, e.g., by He Jifeng and Tony Hoare to model data refinement 131418/27. More fundamentally, saturation can be seen as complementary to the use of laxness rather than as an alternative to it, as we shall explain in Section [6. This reflects the important connection between the theorem proving and problem solving aspects of proof search in LP, as Section 2 further explains. So we would suggest that both approaches are of value, with the interaction between them meriting serious consideration.

Saturation inherently yields a particular kind of compositionality, but one loses the tightness of the relationship between semantic model and operational behaviour. The latter is illustrated by the finiteness of branching in a coinductive tree, in contrast to the infinity of possible substitutions, which are inherent in saturation. To the extent that it is possible, we would like to recover operational semantics from the semantic model, along the lines of [28, requiring maintenance of intensionality where possible. We regard the distinction between ListNat and GC as a positive feature of lax semantics, as a goal of semantics is to shed light on the critical issues of programming, relation of existential programs to theorem-proving in LP being one such [9]. So we regard Section 6 as supporting both lax and saturation semantics, the interaction between them shedding light on logic programming.

\section{Background: theorem proving in LP}

A signature $\Sigma$ consists of a set $\mathcal{F}$ of function symbols $f, g, \ldots$ each equipped with an arity. Nullary (0-ary) function symbols are constants. For any set Var of variables, the set $\operatorname{Ter}(\Sigma)$ of terms over $\Sigma$ is defined inductively as usual:

$-x \in \operatorname{Ter}(\Sigma)$ for every $x \in \operatorname{Var}$.

- If $f$ is an n-ary function symbol $(n \geq 0)$ and $t_{1}, \ldots, t_{n} \in \operatorname{Ter}(\Sigma)$, then $f\left(t_{1}, \ldots, t_{n}\right) \in \operatorname{Ter}(\Sigma)$.

A substitution over $\Sigma$ is a total function $\sigma: \operatorname{Var} \rightarrow \operatorname{Term}(\Sigma)$. Substitutions are extended from variables to terms as usual: if $t \in \operatorname{Term}(\Sigma)$ and $\sigma$ is a substitution, then the application $\sigma(t)$ is a result of applying $\sigma$ to all variables in $t$. A substitution $\sigma$ is a unifier for $t, u$ if $\sigma(t)=\sigma(u)$, and is a matcher for $t$ against $u$ if $\sigma(t)=u$. A substitution $\sigma$ is a most general unifier (mgu) for $t$ and $u$ if it is a unifier for $t$ and $u$ and is more general than any other such unifier. A most general matcher (mgm) $\sigma$ for $t$ against $u$ is defined analogously.

In line with LP tradition [25, we also take a set $\mathcal{P}$ of predicate symbols each equipped with an arity. It is possible to define logic programs over terms only, in 
line with term-rewriting (TRS) tradition [33, as we do in [15, but we will follow the usual LP tradition here. This gives us the following inductive definitions of the sets of atomic formulae, Horn clauses and logic programs (we also include the definition of terms here for convenience):

\section{Definition 1.}

Terms Ter ::= Var $\mid \mathcal{F}($ Ter,$\ldots$, Ter $)$

Atomic formulae (or atoms) At ::= $\mathcal{P}(T e r, \ldots, T e r)$

(Horn) clauses $H C::=A t \leftarrow A t, \ldots, A t$

Logic programs Prog $::=H C, \ldots, H C$

In what follows, we will use letters $A, B, C, D$, possibly with subscripts, to refer to elements of $A t$.

Given a logic program $P$, we may ask whether a certain atom is logically entailed by $P$. E.g., given the program ListNat we may ask whether list (cons $(0$, nil $))$ is entailed by ListNat. The following rule, which is a restricted form of the famous SLD-resolution, provides a semi-decision procedure to derive the entailment:

\section{Definition 2 (Term-matching (TM) Resolution).}

$$
\overline{P \vdash[]} \quad \frac{P \vdash \sigma A_{1} \quad \cdots \quad P \vdash \sigma A_{n}}{P \vdash \sigma A} \text { if }\left(A \leftarrow A_{1}, \ldots, A_{n}\right) \in P
$$

In contrast, the SLD-resolution rule could be presented in the following form:

$$
B_{1}, \ldots, B_{j}, \ldots, B_{n} \leadsto P \sigma B_{1}, \ldots, \sigma A_{1}, \ldots, \sigma A_{n}, \ldots, \sigma B_{n},
$$

if $\left(A \leftarrow A_{1}, \ldots, A_{n}\right) \in P$, and $\sigma$ is the mgu of $A$ and $B_{j}$. The derivation for $A$ succeeds when $A \sim_{P}$ []; we use $\sim_{P}^{*}$ to denote several steps of SLD-resolution.

At first sight, the difference between TM-resolution and SLD-resolution seems to be that of notation. Indeed, both ListNat $\vdash$ list $(\operatorname{cons}(0, \mathrm{nil}))$ and

list $(\operatorname{cons}(0, \mathrm{nil})) \sim_{\text {List Nat }}^{*}$ [] by the above rules (see also Figure 1). However, ListNat $\nvdash \operatorname{list}(\operatorname{cons}(\mathrm{x}, \mathrm{y}))$ whereas $\operatorname{list}(\operatorname{cons}(\mathrm{x}, \mathrm{y})) \sim_{\text {ListNat }}^{*}[]$. And, even more mysteriously, $G C \nvdash$ connected(x,y) while connected(x,y) $\sim_{G C}$ [ ].

As it turns out, TM-resolution reflects the theorem proving side of LP: rules of Definition 2 can be used to semi-decide whether a given term $t$ is entailed by $P$. In contrast, SLD-resolution reflects the problem solving aspect of LP: using the SLD-resolution rule, one asks whether, for a given $t$, a substitution $\sigma$ can be found such that $P \vdash \sigma(t)$. There is a subtle but important difference between these two aspects of proof search.

For example, when considering the successful derivation list $(\operatorname{cons}(\mathrm{x}, \mathrm{y}))$ $\sim_{\text {ListNat }}^{*}[]$, we assume that list(cons $\left.(\mathrm{x}, \mathrm{y})\right)$ holds only relative to a computed substitution, e.g. $\mathrm{x} \mapsto 0, \mathrm{y} \mapsto \mathrm{nil}$. Of course this distinction is natural from the point of view of theorem proving: $\operatorname{list}(\operatorname{cons}(\mathrm{x}, \mathrm{y}))$ is not a "theorem" in this generality, but its special case, list $(\operatorname{cons}(0, \mathrm{nil}))$, is. Thus, ListNat $\vdash$ list(cons(0,nil)) but ListNat $\nvdash$ list(cons(x,y)) (see also Figure 1). Similarly, connected $(\mathrm{x}, \mathrm{y}) \sim_{G C}$ [] should be read as: connected $(\mathrm{x}, \mathrm{y})$ holds relative to the computed substitution $\mathrm{y} \mapsto \mathrm{x}$. 
According to the soundness and completeness theorems for SLD-resolution [25], the derivation $\leadsto$ has existential meaning, i.e. when list (cons $(\mathrm{x}, \mathrm{y})) \sim_{\text {ListNat }}^{*}$ [ ], the succeeded goal list $(\operatorname{cons}(\mathrm{x}, \mathrm{y}))$ is not meant to be read as universally quantified over $\mathrm{x}$ an $\mathrm{y}$. On the contrary, TM-resolution proves a universal statement. That is, $G C \vdash \operatorname{connected}(\mathrm{x}, \mathrm{x})$ reads as: connected $(\mathrm{x}, \mathrm{x})$ is entailed by GC for any $\mathrm{x}$.

Much of our recent work has been devoted to formal understanding of the relation between the theorem proving and problem solving aspects of LP [1519. The type-theoretic semantics of TM-resolution, given by "Horn clauses as types, $\lambda$-terms as proofs" is given in 910].

Definition 2 gives rise to derivation trees. E.g. the derivation (or, equivalently, the proof) for ListNat $\vdash$ list(cons $(0, \mathrm{nil}))$ can be represented by the following derivation tree:

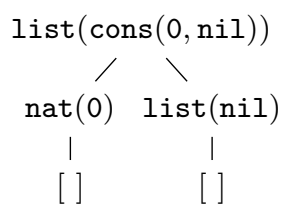

In general, given a term $t$ and a program $P$, more than one derivation for $P \vdash t$ is possible. For example, if we add a fifth clause to program ListNat:

5. list $(\operatorname{cons}(0, x)) \leftarrow \operatorname{list}(x)$

then yet another, alternative, proof is possible for the extended program: List Nat ${ }^{+} \vdash$ list $(\operatorname{cons}(0, \mathrm{nil}))$ via the clause 5 :

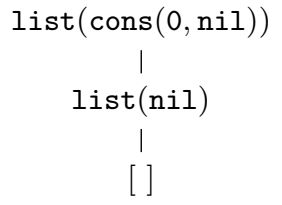

To reflect the choice of derivation strategies at every stage of the derivation, we can introduce a new kind of nodes, or-nodes. For our example, this would give us the tree shown in Figure 1, note the $\bullet$-nodes.

This intuition is made precise in the following definition of a coinductive tree, which first appeared in 21/23] and was later refined in [15] under the name of a rewriting tree. Note the use of mgms (rather than mgus) in the last item.

Definition 3 (Coinductive tree). Let $P$ be a logic program and $A$ be an atomic formula. The coinductive tree for $A$ is the possibly infinite tree $T$ satisfying the following properties.

- $A$ is the root of $T$

- Each node in $T$ is either an and-node or an or-node

- Each or-node is given by $\bullet$

- Each and-node is an atom

- For every and-node $A^{\prime}$ occurring in $T$, if there exist exactly $m>0$ distinct clauses $C_{1}, \ldots, C_{m}$ in $P$ (a clause $C_{i}$ has the form $B_{i} \leftarrow B_{1}^{i}, \ldots, B_{n_{i}}^{i}$ for 

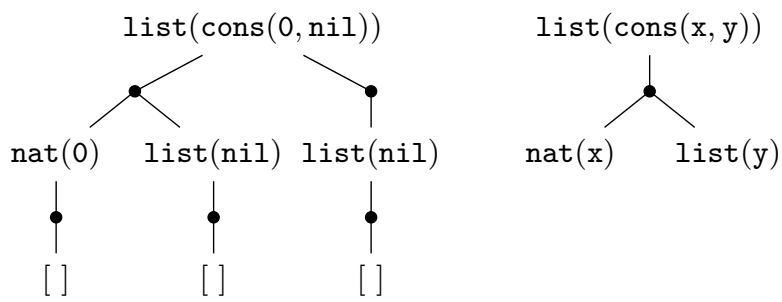

Fig. 1. Left: a coinductive tree for $\operatorname{list}(\operatorname{cons}(0, \mathrm{nil}))$ and the extended program List Nat ${ }^{+}$. Right: a coinductive tree for list $(\operatorname{cons}(\mathrm{x}, \mathrm{y}))$ and $\operatorname{ListNat}^{+}$. The $\bullet$-nodes mark different clauses applicable to every atom in the tree.

some $\left.n_{i}\right)$, such that $A^{\prime}=B_{1} \theta_{1}=\ldots=B_{m} \theta_{m}$, for mgms $\theta_{1}, \ldots, \theta_{m}$, then $A^{\prime}$ has exactly $m$ children given by or-nodes, such that, for every $i \in m$, the $i$-th or-node has $n_{i}$ children given by and-nodes $B_{1}^{i} \theta_{i}, \ldots, B_{n_{i}}^{i} \theta_{i}$.

Coinductive trees provide a convenient model for proofs by TM-resolution.

Let us make one final observation on TM-resolution. Generally, given a program $P$ and an atom $t$, one can prove that

$$
t \sim_{P}^{*}[] \text { with computed substitution } \sigma \text { iff } P \vdash \sigma t \text {. }
$$

This simple fact may give an impression that proofs (and corresponding coinductive trees) for TM-resolution are in some sense fragments of reductions by SLD-resolution. Compare e.g. the right-hand tree of Figure1 before substitution and a grown left-hand tree obtained after the substitution. In this case, we could emulate the problem solving aspect of SLD-resolution by using coinductive trees and allowing to apply substitutions within coinductive trees, as was proposed in 22159]. Such intuition would hold perfectly for e.g. ListNat, but would not hold for existential programs: although there is a one step SLD-derivation for connected $(\mathrm{x}, \mathrm{y}) \sim G C[]($ with $\mathrm{y} \mapsto \mathrm{x})$, TM-resolution proof for $\operatorname{connected}(\mathrm{x}, \mathrm{y})$ diverges and gives rise to the following infinite coinductive tree:

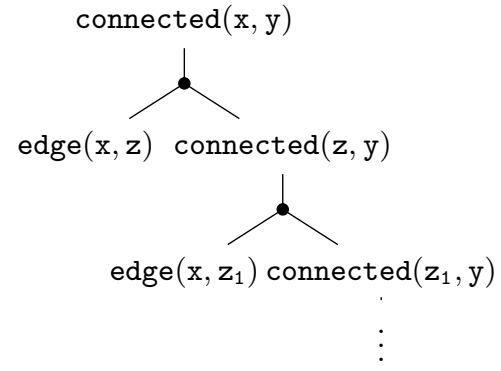

Not only the proof for $G C \vdash \operatorname{connected}(\mathrm{x}, \mathrm{y})$ is not in any sense a fragment of the derivation connected $(\mathrm{x}, \mathrm{y}) \sim_{G C}$ [ ], but it also takes larger (i.e. infinite) signature. Thus, operational semantics of TM-resolution and SLD-resolution can 
be very different for existential programs: both in aspects of termination and signature size.

This problem is orthogonal to non-termination. Consider the non-terminating (but not existential) program Bad:

$\operatorname{bad}(\mathrm{x}) \leftarrow \operatorname{bad}(\mathrm{x})$

For Bad, operational behavior of TM-resolution and SLD-resolution are similar: derivations with both do not terminate and require finite signature. Once again, such programs can be analysed using similar coinductive methods in TM- and SLD-resolution [1030.

The problems caused by existential variables are known in the literature on theorem proving and term-rewriting [33. In TRS [33, existential variables are not allowed to appear in rewriting rules, and in type inference based on term rewriting or TM-resolution, the restriction to non-existential programs is common [11].

So theorem-proving, in contrast to problem-solving, is modelled by termmatching; term-matching gives rise to coinductive trees; and as explained in the introduction and, in more detail, later, coinductive trees give rise to laxness. So in this paper, we use laxness to model coinductive trees, and thereby theoremproving in LP, and relate our semantics with Bonchi and Zanasi's work, which we believe models primarily problem-solving aspect of logic programming.

Categorical semantics for existential programs, which are known to be challenging for theorem proving, is the main contribution of Section 5 and this paper.

\section{Modelling coinductive trees for variable-free logic programs}

In this section, we recall and develop the work of [20] and in particular we restrict our semantics to variable-free logic programs, i.e. we take $V a r=\emptyset$ in Definition 1. Variable-free logic programs are operationally equivalent to propositional logic programs, as substitutions play no role in derivations. In this (propositional) setting, coinductive trees coincide with the and-or derivation trees known in the LP literature [12].

Proposition 1. For any set At, there is a bijection between the set of variablefree logic programs over the set of atoms At and the set of $P_{f} P_{f}$-coalgebra structures on At, where $P_{f}$ is the finite powerset functor on Set.

Theorem 1. Let $C\left(P_{f} P_{f}\right)$ denote the cofree comonad on $P_{f} P_{f}$. Then, for $p$ : $A t \longrightarrow P_{f} P_{f}(A t)$, the corresponding $C\left(P_{f} P_{f}\right)$-coalgebra $\bar{p}: A t \longrightarrow C\left(P_{f} P_{f}\right)(A t)$ sends an atom $A$ to the coinductive tree for $A$.

Proof. Applying the work of 34 to this setting, the cofree comonad is in general determined as follows: $C\left(P_{f} P_{f}\right)(\mathrm{At})$ is the limit of the diagram

$$
\ldots \longrightarrow \text { At } \times P_{f} P_{f}\left(\text { At } \times P_{f} P_{f}(\mathrm{At})\right) \longrightarrow \text { At } \times P_{f} P_{f}(\mathrm{At}) \longrightarrow \text { At. }
$$


with maps determined by the projection $\pi_{0}: A t \times P_{f} P_{f}(A t) \longrightarrow A t$, with applications of the functor $A t \times P_{f} P_{f}(-)$ to it.

Putting $\mathrm{At}_{0}=\mathrm{At}$ and $\mathrm{At}_{n+1}=\mathrm{At} \times P_{f} P_{f} \mathrm{At}_{n}$, and defining the cone

$$
\begin{aligned}
p_{0} & =i d: \mathrm{At} \longrightarrow \mathrm{At}\left(=\mathrm{At}_{0}\right) \\
p_{n+1} & =\left\langle i d, P_{f} P_{f}\left(p_{n}\right) \circ p\right\rangle: \mathrm{At} \longrightarrow \mathrm{At} \times P_{f} P_{f} \mathrm{At}_{n}\left(=\mathrm{At}_{n+1}\right)
\end{aligned}
$$

the limiting property of the diagram determines the coalgebra $\bar{p}:$ At $\longrightarrow$ $C\left(P_{f} P_{f}\right)(\mathrm{At})$. The image $\bar{p}(A)$ of an atom $A$ is given by an element of the limit, equivalently a map from 1 into the limit, equivalently a cone of the diagram over 1.

To give the latter is equivalent to giving an element $A_{0}$ of $A t$, specifically $p_{0}(A)=A$, together with an element $A_{1}$ of $A t \times P_{f} P_{f}(A t)$, specifically $p_{1}(A)=$ $\left(A, p_{0}(A)\right)=(A, p(A))$, together with an element $A_{2}$ of $A t \times P_{f} P_{f}\left(A t \times P_{f} P_{f}(A t)\right)$, etcetera. The definition of the coinductive tree for $A$ is inherently coinductive, matching the definition of the limit, and with the first step agreeing with the definition of $p$. Thus it follows by coinduction that $\bar{p}(A)$ can be identified with the coinductive tree for $A$.

Example 3. Let At consist of atoms A, B, C and D. Let $P$ denote the logic program

$$
\begin{aligned}
& \mathrm{A} \leftarrow \mathrm{B}, \mathrm{C} \\
& \mathrm{A} \leftarrow \mathrm{B}, \mathrm{D} \\
& \mathrm{D} \leftarrow \mathrm{A}, \mathrm{C}
\end{aligned}
$$

So $p(\mathrm{~A})=\{\{\mathrm{B}, \mathrm{C}\},\{\mathrm{B}, \mathrm{D}\}\}, p(\mathrm{~B})=p(\mathrm{C})=\emptyset$, and $p(\mathrm{D})=\{\{\mathrm{A}, \mathrm{C}\}\}$.

Then $p_{0}(\mathrm{~A})=\mathrm{A}$, which is the root of the coinductive tree for $\mathrm{A}$.

Then $p_{1}(\mathrm{~A})=(\mathrm{A}, p(\mathrm{~A}))=(\mathrm{A},\{\{\mathrm{B}, \mathrm{C}\},\{\mathrm{B}, \mathrm{D}\}\})$, which consists of the same information as in the first three levels of the coinductive tree for $A$, i.e., the root A, two or-nodes, and below each of the two or-nodes, nodes given by each atom in each antecedent of each clause with head $\mathrm{A}$ in the logic program $P$ : nodes marked B and C lie below the first or-node, and nodes marked B and D lie below the second or-node, exactly as $p_{1}(\mathrm{~A})$ describes.

Continuing, note that $p_{1}(\mathrm{D})=(\mathrm{D}, p(\mathrm{D}))=(\mathrm{D},\{\{\mathrm{A}, \mathrm{C}\}\})$. So

$$
\begin{aligned}
p_{2}(\mathrm{~A}) & =\left(\mathrm{A}, P_{f} P_{f}\left(p_{1}\right)(p(\mathrm{~A}))\right) \\
& =\left(\mathrm{A}, P_{f} P_{f}\left(p_{1}\right)(\{\{\mathrm{B}, \mathrm{C}\},\{\mathrm{B}, \mathrm{D}\}\})\right) \\
& =(\mathrm{A},\{\{(\mathrm{B}, \emptyset),(\mathrm{C}, \emptyset)\},\{(\mathrm{B}, \emptyset),(\mathrm{D},\{\{\mathrm{A}, \mathrm{C}\}\})\}\})
\end{aligned}
$$

which is the same information as that in the first five levels of the coinductive tree for $\mathrm{A}: p_{1}(\mathrm{~A})$ provides the first three levels of $p_{2}(\mathrm{~A})$ because $p_{2}(\mathrm{~A})$ must map to $p_{1}(\mathrm{~A})$ in the cone; in the coinductive tree, there are two and-nodes at level 3, labelled by A and C. As there are no clauses with head B or C, no or-nodes lie below the first three of the and-nodes at level 3. However, there is one or-node lying below $\mathrm{D}$, it branches into and-nodes labelled by A and C, which is exactly as $p_{2}(\mathrm{~A})$ tells us.

For pictures of such trees, see [23]. 


\section{Modelling coinductive trees for logic programs without existential variables}

We now lift the restriction on $\operatorname{Var}=\emptyset$ in Definition 1 , and consider first-order terms and atoms in full generality, however, we restrict the definition of clauses in Definition 1 to those not containing existential variables.

The Lawvere theory $\mathcal{L}_{\Sigma}$ generated by a signature $\Sigma$ is (up to isomorphism, as there are several equivalent formulations) the category defined as follows: $\mathrm{ob}\left(\mathcal{L}_{\Sigma}\right)$ is the set of natural numbers. For each natural number $n$, let $x_{1}, \ldots, x_{n}$ be a specified list of distinct variables. Define $\mathcal{L}_{\Sigma}(n, m)$ to be the set of $m$ tuples $\left(t_{1}, \ldots, t_{m}\right)$ of terms generated by the function symbols in $\Sigma$ and variables $x_{1}, \ldots, x_{n}$. Define composition in $\mathcal{L}_{\Sigma}$ by substitution.

One can readily check that these constructions satisfy the axioms for a category, with $\mathcal{L}_{\Sigma}$ having strictly associative finite products given by the sum of natural numbers. The terminal object of $\mathcal{L}_{\Sigma}$ is the natural number 0 .

Example 4. Consider ListNat. The constants 0 and nil are maps from 0 to 1 in $\mathcal{L}_{\Sigma}, \mathrm{s}$ is modelled by a map from 1 to 1 , and cons is modelled by a map from 2 to 1 . The term $\mathbf{s}(0)$ is the map from 0 to 1 given by the composite of the maps modelling $\mathrm{s}$ and 0 .

Given an arbitrary logic program $P$ with signature $\Sigma$, we can extend the set $A t$ of atoms for a variable-free logic program to the functor At: $\mathcal{L}_{\Sigma}^{o p} \rightarrow$ Set that sends a natural number $n$ to the set of all atomic formulae, with variables among $x_{1}, \ldots, x_{n}$, generated by the function symbols in $\Sigma$ and by the predicate symbols in $P$. A map $f: n \rightarrow m$ in $\mathcal{L}_{\Sigma}$ is sent to the function $A t(f): A t(m) \rightarrow A t(n)$ that sends an atomic formula $A\left(x_{1}, \ldots, x_{m}\right)$ to $A\left(f_{1}\left(x_{1}, \ldots, x_{n}\right) / x_{1}, \ldots, f_{m}\left(x_{1}, \ldots, x_{n}\right) / x_{m}\right)$, i.e., $A t(f)$ is defined by substitution.

As explained in the Introduction and in 20, we cannot model a logic program by a natural transformation of the form $p: A t \longrightarrow P_{f} P_{f} A t$ as naturality breaks down, e.g., in ListNat. So, in 2123, we relaxed naturality to lax naturality. In order to define it, we extended $A t: \mathcal{L}_{\Sigma}^{o p} \rightarrow$ Set to have codomain Poset by composing At with the inclusion of Set into Poset. Mildly overloading notation, we denote the composite by $A t: \mathcal{L}_{\Sigma}^{o p} \rightarrow$ Poset.

Definition 4. Given functors $H, K: \mathcal{L}_{\Sigma}^{o p} \longrightarrow$ Poset, a lax transformation from $H$ to $K$ is the assignment to each object $n$ of $\mathcal{L}_{\Sigma}$, of an order-preserving function $\alpha_{n}: H n \longrightarrow K n$ such that for each map $f: n \longrightarrow m$ in $\mathcal{L}_{\Sigma}$, one has $(K f)\left(\alpha_{m}\right) \leq\left(\alpha_{n}\right)(H f)$, pictured as follows:

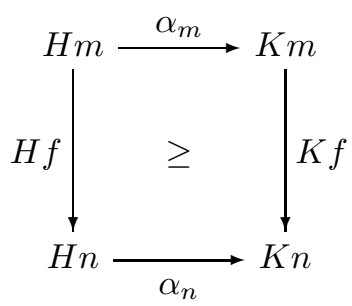


Functors and lax transformations, with pointwise composition, form a locally ordered category denoted by $\operatorname{Lax}\left(\mathcal{L}_{\Sigma}^{o p}\right.$, Poset $)$. Such categories and generalisations have been studied extensively, e.g., in [2]316]18.

Definition 5. Define $P_{f}:$ Poset $\longrightarrow$ Poset by letting $P_{f}(P)$ be the partial order given by the set of finite subsets of $P$, with $A \leq B$ if for all $a \in A$, there exists $b \in B$ for which $a \leq b$ in $P$, with behaviour on maps given by image. Define $P_{c}$ similarly but with countability replacing finiteness.

We are not interested in arbitrary posets in modelling logic programming, only those that arise, albeit inductively, by taking subsets of a set qua discrete poset. So we gloss over the fact that, for an arbitrary poset $P$, Definition 5 may yield factoring, with the underlying set of $P_{f}(P)$ being a quotient of the set of subsets of $P$. It does not affect the line of development here.

Example 5. Modelling Example1 ListNat generates a lax transformation of the form $p: A t \longrightarrow P_{f} P_{f} A t$ as follows: $A t(n)$ is the set of atomic formulae in ListNat with at most $n$ variables.

For example, $A t(0)$ consists of nat(0), nat(nil), list(0), list(nil), nat(s(0)), nat(s(nil)), list(s(0)), list(s(nil)), nat(cons $(0,0))$, nat (cons $(0, \operatorname{nil}))$, nat (cons(nil, 0)), nat(cons(nil, nil)), etcetera.

Similarly, At(1) includes all atomic formulae containing at most one (specified) variable $x$, thus all the elements of $A t(0)$ together with nat $(\mathrm{x})$, list $(\mathrm{x})$, $\operatorname{nat}(\mathrm{s}(\mathrm{x}))$, list $(\mathrm{s}(\mathrm{x})), \operatorname{nat}(\operatorname{cons}(0, \mathrm{x}))$, nat $(\operatorname{cons}(\mathrm{x}, 0))$, nat $(\operatorname{cons}(\mathrm{x}, \mathrm{x}))$, etcetera.

The function $p_{n}: A t(n) \longrightarrow P_{f} P_{f} A t(n)$ sends each element of $A t(n)$, i.e., each atom $A\left(x_{1}, \ldots, x_{n}\right)$ with variables among $x_{1}, \ldots, x_{n}$, to the set of sets of atoms in the antecedent of each unifying substituted instance of a clause in $P$ with head for which a unifying substitution agrees with $A\left(x_{1}, \ldots, x_{n}\right)$.

Taking $n=0$, nat $(0) \in A t(0)$ is the head of one clause, and there is no other clause for which a unifying substitution will make its head agree with nat(0). The clause with head nat(0) has the empty set of atoms as its tail, so $p_{0}(\operatorname{nat}(0))=\{\emptyset\}$.

Taking $n=1$, list $(\operatorname{cons}(\mathrm{x}, 0)) \in A t(1)$ is the head of one clause given by a unifying substititution applied to the final clause of ListNat, and accordingly $p_{1}(\operatorname{list}(\operatorname{cons}(\mathrm{x}, 0)))=\{\{\operatorname{nat}(\mathrm{x}), \operatorname{list}(0)\}\}$.

The family of functions $p_{n}$ satisfy the inequality required to form a lax transformation precisely because of the allowability of substitution instances of clauses, as in turn is required to model logic programming. The family does not satisfy the strict requirement of naturality as explained in the introduction.

Example 6. Attempting to model Example2 by mimicking the model of ListNat as a lax transformation of the form $p: A t \longrightarrow P_{f} P_{f} A t$ in Example 5 fails.

Consider the clause

$$
\text { connected }(x, y) \leftarrow \operatorname{edge}(x, z), \text { connected }(z, y)
$$

Modulo possible renaming of variables, the head of the clause, i.e., the atom connected(x,y), lies in $A t(2)$ as it has two variables. However, the tail does not lie in $P_{f} P_{f} A t(2)$ as the tail has three variables rather than two. 
We dealt with that inelegantly in [21]: in order to allow $p_{2}($ connected $(\mathrm{x}, \mathrm{y}))$ to model GC in any reasonable sense, we allowed substitutions for $z$ by any term on $x, y$ on the basis that there is no unifying such, so we had better allow all possibilities. So, rather than modelling the clause directly, recalling that $A t(2) \subseteq$ $A t(3) \subseteq A t(4)$, etcetera, modulo renaming of variables, we put

$$
\begin{aligned}
p_{2}(\operatorname{connected}(\mathrm{x}, \mathrm{y}))= & \{\{\operatorname{edge}(\mathrm{x}, \mathrm{x}), \operatorname{connected}(\mathrm{x}, \mathrm{y})\},\{\operatorname{edge}(\mathrm{x}, \mathrm{y}), \operatorname{connected}(\mathrm{y}, \mathrm{y})\}\} \\
p_{3}(\operatorname{connected}(\mathrm{x}, \mathrm{y}))= & \{\{\operatorname{edge}(\mathrm{x}, \mathrm{x}), \operatorname{connected}(\mathrm{x}, \mathrm{y})\},\{\operatorname{edge}(\mathrm{x}, \mathrm{y}), \operatorname{connected}(\mathrm{y}, \mathrm{y})\} \\
& \{\text { edge }(\mathrm{x}, \mathrm{z}), \operatorname{connected}(\mathrm{z}, \mathrm{y})\}\} \\
p_{4}(\operatorname{connected}(\mathrm{x}, \mathrm{y}))= & \{\{\operatorname{edge}(\mathrm{x}, \mathrm{x}), \operatorname{connected}(\mathrm{x}, \mathrm{y})\},\{\operatorname{edge}(\mathrm{x}, \mathrm{y}), \operatorname{connected}(\mathrm{y}, \mathrm{y})\}, \\
& \{\text { edge }(\mathrm{x}, \mathrm{z}), \operatorname{connected}(\mathrm{z}, \mathrm{y})\},\{\operatorname{edge}(\mathrm{x}, \mathrm{w}), \operatorname{connected}(\mathrm{w}, \mathrm{y})\}\}
\end{aligned}
$$

etcetera: for $p_{2}$, as only two variables $x$ and $y$ appear in any element of $P_{f} P_{f} A t(2)$, we allowed substitution by either $x$ or $y$ for $z$; for $p_{3}$, a third variable may appear in an element of $P_{f} P_{f} A t(3)$, allowing an additional possible subsitution; for $p_{4}$, a fourth variable may appear, etcetera.

Countability arises if a unary symbol $s$ is added to GC, as in that case, for $p_{2}$, not only did we allow $x$ and $y$ to be substituted for $z$, but we also allowed $s^{n}(x)$ and $s^{n}(y)$ for any $n>0$, and to do that, we replaced $P_{f} P_{f}$ by $P_{c} P_{f}$, allowing for the countably many possible substitutions.

Those were inelegant decisions, but they allowed us to give some kind of model of all logic programs.

We now turn to the relationship between the lax transformation $p: A t \longrightarrow$ $P_{c} P_{f}$ At modelling a logic program $P$ and $\bar{p}: A t \longrightarrow C\left(P_{c} P_{f}\right) A t$, the corresponding coalgebra for the cofree comonad $C\left(P_{c} P_{f}\right)$ on $P_{c} P_{f}$.

We recall the central abstract result of [21, the notion of an "oplax" map of coalgebras being required to match that of lax transformation. Notation of the form $H$-coalg refers to coalgebras for an endofunctor $H$, while notation of the form $C$-Coalg refers to coalgebras for a comonad $C$. The subscript oplax refers to oplax maps, and given an endofunctor $E$ on Poset, the notation $\operatorname{Lax}\left(\mathcal{L}_{\Sigma}^{o p}, E\right)$ denotes the endofunctor on $\operatorname{Lax}\left(\mathcal{L}_{\Sigma}^{o p}\right.$, Poset $)$ given by post-composition with $E$; similarly for a comonad.

Theorem 2. For any locally ordered endofunctor $E$ on Poset, if $C(E)$ is the cofree comonad on $E$, then there is a canonical isomorphism

$$
\operatorname{Lax}\left(\mathcal{L}_{\Sigma}^{o p}, E\right)-\text { coalg }_{\text {oplax }} \simeq \operatorname{Lax}\left(\mathcal{L}_{\Sigma}^{o p}, C(E)\right)-\text { Coalg }_{\text {oplax }}
$$

Corollary 1. $\operatorname{Lax}\left(\mathcal{L}_{\Sigma}^{o p}, C\left(P_{c} P_{f}\right)\right)$ is the cofree comonad on $\operatorname{Lax}\left(\mathcal{L}_{\Sigma}^{o p}, P_{c} P_{f}\right)$.

Corollary 1 gives a bijection between lax transformations

$$
p: A t \longrightarrow P_{c} P_{f} A t
$$

and lax transformations

$$
\bar{p}: A t \longrightarrow C\left(P_{c} P_{f}\right) A t
$$


subject to the two conditions required of a coalgebra of a comonad. Subject to the routine replacement of the outer copy of $P_{f}$ by $P_{c}$ in the construction in Theorem 11 the same construction, if understood pointwise, extends to this setting, i.e., if one uniformly replaces $A t$ by $A t(n)$ in the construction of Theorem 1 and replaces the outer copy of $P_{f}$ by $P_{c}$, one obtains a description of $C\left(P_{c} P_{f}\right) A t(n)$ together with the construction of $\bar{p}_{n}$ from $p_{n}$.

That is fine for ListNat, modelling the coinductive trees generated by ListNat, the same holding for any logic program without existential variables, but for GC, as explained in Example 6, $p$ did not model the clause

$$
\operatorname{connected}(\mathrm{x}, \mathrm{y}) \leftarrow \operatorname{edge}(\mathrm{x}, \mathrm{z}), \operatorname{connected}(\mathrm{z}, \mathrm{y})
$$

directly, and so its extension a fortiori could not model the coinductive trees generated by connected $(\mathrm{x}, \mathrm{y})$.

For arbitrary logic programs, $\bar{p}\left(A\left(x_{1}, \ldots, x_{n}\right)\right)$ was a variant of the coinductive tree generated by $A\left(x_{1}, \ldots, x_{n}\right)$ in two key ways:

1. coinductive trees allow new variables to be introduced as one passes down the tree, e.g., with

$$
\operatorname{connected}(\mathrm{x}, \mathrm{y}) \leftarrow \operatorname{edge}(\mathrm{x}, \mathrm{z}), \operatorname{connected}(\mathrm{z}, \mathrm{y})
$$

appearing directly in it, whereas, extending Example $6 . \overline{p_{1}}(\operatorname{connected}(\mathrm{x}, \mathrm{y}))$ does not model such a clause directly, but rather substitutes terms on $x$ and $y$ for $z$, continuing inductively as one proceeds.

2. coinductive trees are finitely branching, as one expects in logic programming, whereas $\bar{p}\left(A\left(x_{1}, \ldots, x_{n}\right)\right)$ could be infinitely branching, e.g., for GC with an additional unary operation $s$.

\section{Modelling coinductive trees for arbitrary logic progams}

We believe that our work in 21] provides an interesting model of ListNat, in particular because it agrees with the coinductive trees generated by ListNat. However, the account in 21] is less interesting when applied to GC, thus in the full generality of logic programming. Restriction to non-existential examples such as ListNat is common for implementational reasons [23]15|9|10], so [21] does allow the modeling of coinductive trees for a natural class of logic programs. Here we seek to model coinductive trees for logic programs in general, a fortiori doing so for GC.

In order to model coinductive trees, it follows from Example 6 that the endofunctor $\operatorname{Lax}\left(\mathcal{L}_{\Sigma}^{o p}, P_{f} P_{f}\right)$ on $\operatorname{Lax}\left(\mathcal{L}_{\Sigma}^{o p}\right.$, Poset $)$ that sends $A t$ to $P_{f} P_{f} A t$, needs to be refined as $\{\{\operatorname{edge}(\mathrm{x}, \mathbf{z})$, connected $(\mathbf{z}, \mathrm{y})\}\}$ is not an element of $P_{f} P_{f} A t(2)$ as it involves three variables $x, y$ and $z$. Motivated by that example, we refine our axiomatics in general so that the codomain of $p_{n}$ is a superset of $P_{f} P_{f} A t(m)$ for every $m \geq n$. There are six injections of 2 into 3, inducing six inclusions $A t(2) \subseteq A t(3)$, so six inclusions $P_{f} P_{f} A t(2) \subseteq P_{f} P_{f} A t(3)$, and one only wants 
to count each element of $P_{f} P_{f} A t(2)$ once. So we refine $P_{f} P_{f} A t(n)$ to become $\left(\Sigma_{m \geq n} P_{f} P_{f} A t(m)\right) / \equiv$, where $\equiv$ is generated by the injections $i: n \longrightarrow m$. This can be made precise in abstract category theoretic terms as follows.

For any Lawvere theory $L$, there is a canonical identity-on-objects functor from the category $I n j$ of injections $i: n \longrightarrow m$ of natural numbers into $L^{o p}$. So, in particular, there is a canonical identity on objects functor $J: \operatorname{Inj} \longrightarrow \mathcal{L}_{\Sigma}^{o p}$, upon which $\Sigma_{m \geq n} P_{f} P_{f} A t(m) / \equiv$ may be characterised as the colimit (see [26] or, for the enriched version, [17])

$$
\int^{m \in n / I n j} P_{f} P_{f} A t J(m)
$$

or equivalently, given $n \in I n j$, the colimit of the functor from $n / I n j$ to Poset that sends an injection $j: n \longrightarrow m$ to $P_{f} P_{f} A t J(m)$.

This construction extends to a functor $P_{f f}(A t): \mathcal{L}_{\Sigma}^{o p} \longrightarrow$ Poset by sending a map $f: n \longrightarrow n^{\prime}$ in $\mathcal{L}_{\Sigma}$ to the order-preserving function

$$
\int^{m \in n^{\prime} / I n j} P_{f} P_{f} \operatorname{AtJ}(m) \longrightarrow \int^{m \in n / I n j} P_{f} P_{f} \operatorname{AtJ}(m)
$$

determined by the fact that each $m \in n^{\prime} / \operatorname{Inj}$ is, up to coherent isomorphism, uniquely of the form $n^{\prime}+k$, allowing one to apply $P_{f} P_{f} A t$ to the map $f+k$ : $n+k \longrightarrow n^{\prime}+k=m$ in $\mathcal{L}_{\Sigma}$. This is similar to the behaviour of the monad for local state on maps [29].

It is routine to generalise the construction from $A t$ to make it apply to an arbitrary functor $H: \mathcal{L}_{\Sigma}^{o p} \longrightarrow$ Poset.

In order to make the construction functorial, i.e., in order to make it respect maps $\alpha: H \Rightarrow K$, we need to refine $\operatorname{Lax}\left(\mathcal{L}_{\Sigma}^{o p}\right.$, Poset $)$ as the above colimit strictly respects injections, i.e., for any injection $i: n \longrightarrow m$, we want the diagram

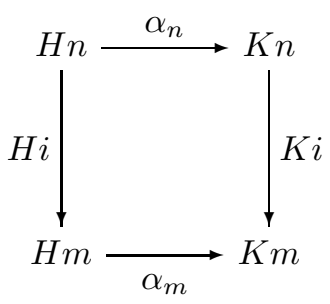

to commute.

Summarising this discussion yields the following:

Definition 6. Let $\operatorname{Lax}_{I n j}\left(\mathcal{L}_{\Sigma}^{o p}\right.$, Poset) denote the category with objects given by functors from $\mathcal{L}_{\Sigma}^{o p}$ to Poset, maps given by lax transformations that strictly respect injections, and composition given pointwise.

Proposition 2. cf [29] Let $J:$ Inj $\longrightarrow \mathcal{L}_{\Sigma}^{o p}$ be the canonical inclusion. Define

$$
P_{f f}: \operatorname{Lax}_{\text {Inj }}\left(\mathcal{L}_{\Sigma}^{o p}, \text { Poset }\right) \longrightarrow \operatorname{Lax}_{\text {Inj }}\left(\mathcal{L}_{\Sigma}^{o p}, \text { Poset }\right)
$$




$$
\begin{gathered}
\text { by }\left(P_{f f}(H)\right)(n)=\int^{m \in n / I n j} P_{f} P_{f} H J(m), \text { with, for any map } f: n \longrightarrow n^{\prime} \text { in } \mathcal{L}_{\Sigma}, \\
\left(P_{f f}(H)\right)(f): \int^{m \in n^{\prime} / I n j} P_{f} P_{f} H J(m) \longrightarrow \int^{m \in n / I n j} P_{f} P_{f} H J(m)
\end{gathered}
$$

determined by the fact that each $m \in n^{\prime} /$ Inj is, up to coherent isomorphism, uniquely of the form $n^{\prime}+k$, allowing one to apply $P_{f} P_{f} H$ to the map $f+k$ : $n+k \longrightarrow n^{\prime}+k=m$ in $\mathcal{L}_{\Sigma}$.

Given $\alpha: H \Rightarrow K$, define $P_{f f}(\alpha)(n)$ by the fact that $m \in n /$ Inj is uniquely of the form $n+k$, and using

$$
\alpha_{n+k}: H(m)=H(n+k) \longrightarrow K(n+k)=K(m)
$$

Then $P_{f f}$ is an endofunctor on $\operatorname{Lax}_{I n j}\left(\mathcal{L}_{\Sigma}^{o p}\right.$, Poset $)$.

The proof is routine but requires lengthy calculation involving colimits. Observe that we have not required countability anywhere in the definition of $P_{f f}$, using only finiteness as we sought at the end of Section 4.

We can now model an arbitrary logic program by a map $p: A t \longrightarrow P_{f f} A t$ in $\operatorname{Lax}_{\text {Inj }}\left(\mathcal{L}_{\Sigma}^{o p}\right.$, Poset $)$, modelling ListNat as we did in Example 5 but now modelling the clauses of GC directly rather than using the awkward substitution instances of Example 6 .

Example 7. Except for the restriction of $\operatorname{Lax}\left(\mathcal{L}_{\Sigma}^{o p}\right.$, Poset $)$ to $\operatorname{Lax}{ }_{I n j}\left(\mathcal{L}_{\Sigma}^{o p}\right.$, Poset $)$, ListNat is modelled in exactly the same way here as it was in Example 5 , the reason being that no clause in ListNat has a variable in the tail that does not already appear in the head. We need only observe that, although $p$ is not strictly natural in general, it does strictly respect injections. For example, if one views list(cons $(\mathrm{x}, 0))$ as an element of $A t(2)$, its image under $p_{2}$ agrees with its image under $p_{1}$.

Example 8. In contrast to Example 6, using $P_{f f}$, we can emulate the construction of Examples 5 and 7 for ListNat to model GC.

Modulo possible renaming of variables, connected(x,y) is an element of $A t(2)$. The function $p_{2}$ sends it to the element $\{\{\operatorname{edge}(\mathrm{x}, \mathrm{z}), \operatorname{connected}(\mathrm{z}, \mathrm{y})\}\}$ of $\left(P_{f f}(A t)\right)(2)$. This is possible by taking $n=2$ and $m=3$ in the formula for $P_{f f}(A t)$ in Proposition 2, In contrast, $\{\{$ edge $(\mathrm{x}, \mathrm{z})$, connected $(\mathrm{z}, \mathrm{y})\}\}$ is not an element of $P_{f} P_{f} A t(2)$, hence the failure of Example 6 .

The behaviour of $P_{f f}(A t)$ on maps ensures that the lax transformation $p$ strictly respects injections. For example, if connected $(\mathrm{x}, \mathrm{y})$ is seen as an element of $A t(3)$, the additional variable is treated as a fresh variable $w$, so does not affect the image of connected $(\mathrm{x}, \mathrm{y})$ under $p_{3}$.

Theorem 3. The functor $P_{f f}: \operatorname{Lax}_{I n j}\left(\mathcal{L}_{\Sigma}^{o p}\right.$, Poset $) \longrightarrow \operatorname{Lax}_{I n j}\left(\mathcal{L}_{\Sigma}^{\text {op }}\right.$, Poset $)$ induces a cofree comonad $C\left(P_{f f}\right)$ on $\operatorname{Lax}_{I n j}\left(\mathcal{L}_{\Sigma}^{o p}\right.$, Poset $)$. Moreover, given a logic progam $P$ qua $P_{f f}$-coalgebra $p: A t \longrightarrow P_{f f}(A t)$, the corresponding $C\left(P_{f f}\right)$ coalgebra $\bar{p}: A t \longrightarrow C\left(P_{f f}\right)(A t)$ sends an atom $A\left(x_{1}, \ldots, x_{n}\right) \in A t(n)$ to the coinductive tree for $A\left(x_{1}, \ldots, x_{n}\right)$. 
Proof. The construction of Theorem 1 subject to mild rephrasing, continues to work here. Specifically, $\left(C\left(P_{f f}\right) A t\right)(n)$ is given by the same limit as in Theorem 1 but with $A t$ replaced by $A t(n)$ and with $P_{f} P_{f}$ replaced by $P_{f f}$ : products in the category $\operatorname{Lax}_{I n j}\left(\mathcal{L}_{\Sigma}^{o p}\right.$, Poset $)$ are given pointwise, so the use of projections is the same; $[$ Inj, Poset $]$ is locally finitely presentable and $P_{f f}$ is an accessible functor, allowing us to extend the construction of the cofree comonad pointwise to $\left[\right.$ Inj, Poset]. It is routine, albeit tedious, to verify functoriality of $C\left(P_{f f}\right)$ with respect to all maps and to verify the universal property. The construction of $\bar{p}$ is given pointwise, with it following from its coinductive construction that it yields the coinductive trees as required.

The lax naturality in respect to general maps $f: m \longrightarrow n$ means that a substitution applied to an atom $A\left(x_{1}, \ldots, x_{n}\right) \in A t(n)$, i.e., application of the function $A t(f)$ to $A\left(x_{1}, \ldots, x_{n}\right)$, followed by application of $\bar{p}$, i.e., taking the coinductive tree for the substituted atom, or application of the function $\left.\left(C\left(P_{f} f\right) A t\right) f\right)$ to the coinductive tree for $A\left(x_{1}, \ldots, x_{n}\right)$ potentially yield different trees: the former substitutes into $A\left(x_{1}, \ldots, x_{n}\right)$, then takes its coinductive tree, while the latter applies a substitution to each node of the coinductive tree for $A\left(x_{1}, \ldots, x_{n}\right)$, then prunes to remove redundant branches.

Example 9. Extending Example 8, consider connected(x,y) $\in A t(2)$. In expressing GC as a map $p: A t \longrightarrow P_{f f} A t$ in Example 8 , we put

$$
p_{2}(\operatorname{connected}(\mathrm{x}, \mathrm{y}))=\{\{\operatorname{edge}(\mathrm{x}, \mathrm{z}), \operatorname{connected}(\mathrm{z}, \mathrm{y})\}\}
$$

Accordingly, $\bar{p}_{2}(\operatorname{connected}(\mathrm{x}, \mathrm{y}))$ is the coinductive tree for connected( $\left.\mathrm{x}, \mathrm{y}\right)$, thus the infinite tree generated by repeated application of the same clause modulo renaming of variables.

If we substitute $x$ for $y$ in the coinductive tree, i.e., apply the function $\left(C\left(P_{f f}\right) A t\right)(x, x)$ to it (see the definition of $L_{\Sigma}$ at the start of Section 4 and observe that $(x, x)$ is a 2 -tuple of terms generated trivially by the variable $x$ ), we obtain the same tree but with $y$ systematically replaced by $x$. However, if we substitute $x$ for $y$ in connected(x,y), i.e., apply the function $A t(x, x)$ to it, we obtain connected $(\mathrm{x}, \mathrm{x}) \in A t(1)$, whose coinductive tree has additional branching as the first clause of GC, i.e., connected $(\mathrm{x}, \mathrm{x}) \leftarrow$ may also be applied.

In contrast to this, we have strict naturality with respect to injections: for example, an injection $i: 2 \longrightarrow 3$ yields the function $A t(i): A t(2) \longrightarrow A t(3)$ that, modulo renaming of variables, sends connected(x,y) $\in A t(2)$ to itself seen as an element of $A t(3)$, and the coinductive tree for connected $(\mathrm{x}, \mathrm{y})$ is accordingly also sent by $\left(C\left(P_{f f}\right) A t\right)(i)$ to itself seen as an element of $\left(C\left(P_{f f}\right) A t\right)(3)$.

Example 9 illustrates why, although the condition of strict naturality with respect to injections holds for $P_{f f}$, it does not hold for $\operatorname{Lax}\left(\mathcal{L}_{\Sigma}^{o p}, P_{f} P_{f}\right)$ in Example 6 as we did not model the clause

$$
\text { connected }(\mathrm{x}, \mathrm{y}) \leftarrow \operatorname{edge}(\mathrm{x}, \mathrm{z}), \text { connected }(\mathrm{z}, \mathrm{y})
$$

directly there, but rather modelled all substitution instances into all available variables. 


\section{Complementing saturated semantics}

Bonchi and Zanasi's approach to modelling logic programming in 6] was to consider $P_{f} P_{f}$ as we did in [21], sending $A t$ to $P_{f} P_{f} A t$, but to ignore the inherent laxness, replacing $\operatorname{Lax}\left(\mathcal{L}_{\Sigma}^{o p}\right.$, Poset $)$ by $\left[o b\left(\mathcal{L}_{\Sigma}\right), \operatorname{Set}\right]$, where $o b\left(\mathcal{L}_{\Sigma}\right)$ is the set of objects of $\mathcal{L}_{\Sigma}$ treated as a discrete category, i.e., as one with only identity maps.

The central mathematical fact that supports saturated semantics is that, regarding $o b\left(\mathcal{L}_{\Sigma}\right)$ as a discrete category, with inclusion functor $I: o b\left(\mathcal{L}_{\Sigma}\right) \longrightarrow$ $\mathcal{L}_{\Sigma}$, the functor

$$
[I, S e t]:\left[\mathcal{L}_{\Sigma}^{o p}, S e t\right] \longrightarrow\left[o b\left(\mathcal{L}_{\Sigma}\right)^{o p}, S e t\right]
$$

that sends a functor $H: \mathcal{L}_{\Sigma}^{o p} \longrightarrow$ Set to the composite functor $H I: o b\left(\mathcal{L}_{\Sigma}\right)=$ $o b\left(\mathcal{L}_{\Sigma}\right)^{o p} \longrightarrow$ Set has a right adjoint. That adjoint is given by right Kan extension. It is primarily the fact of the existence of the right adjoint, rather than its characterisation as a right Kan extension, that enabled Bonchi and Zanasi's various constructions, in particular those of saturation and desaturation.

That allows us to mimic Bonchi and Zanasi's saturation semantics, but starting from $\operatorname{Lax}\left(\mathcal{L}_{\Sigma}^{o p}\right.$, Poset $)$ rather than from $\left[o b\left(\mathcal{L}_{\Sigma}\right), S e t\right]$. We are keen to allow this as laxness is an inherent fact of the situation, as we have explained through the course of this paper. Such laxness has been valuable in related semantic endeavours, such as in Tony Hoare's pioneering work on the modelling of data refinement 13 14 18, of which substitution in logic programming can be seen as an instance.

The argument, which was originally due to Ross Street, cf [32, goes as follows.

Theorem 4. [3] For any finitary 2-monad $T$ on a cocomplete 2-category $K$, the inclusion

$$
J: T-A l g_{s} \longrightarrow T-A l g_{l}
$$

of the category of strict $T$-algebras and strict maps of T-algebras into the category of strict T-algebras and lax maps of T-algebras has a left adjoint.

Example 10. For any Lawvere theory $L$, there is a finitary locally ordered monad $T$ on $\left[o b(L)\right.$, Poset $\left.^{o p}\right]$ for which $\left[L\right.$, Poset $\left.^{o p}\right]$ is isomorphic to $T$-Alg $g_{s}$, with $T$ - $A l g_{l}$ isomorphic to $\operatorname{Lax}\left(L\right.$, Poset $\left.^{o p}\right)$. The monad $T$ is given by the composite of the functor

$$
\left[J, \text { Poset }^{o p}\right]:\left[L, \text { Poset }^{o p}\right] \longrightarrow\left[o b(L), \text { Poset }^{o p}\right]
$$

where $J: o b(L) \longrightarrow L$ is the inclusion, cf Bonchi and Zanasi's construction [6], with its left adjoint, which is given by left Kan extension. The fact that the functor $\left[J\right.$, Poset $\left.^{o p}\right]$ also has a right adjoint, given by right Kan extension, implies that the monad $T$ is finitary.

Corollary 2. For any Lawvere theory L, the inclusion

$$
\left[L^{o p}, \text { Poset }\right] \longrightarrow \operatorname{Lax}\left(L^{o p}, \text { Poset }\right)
$$

has a right adjoint. 
Proof. Poset is a complete 2-category as it is a complete locally ordered category. So Poset ${ }^{o p}$ is a cocomplete 2-category, and so $\left[o b(L)\right.$, Poset $\left.^{o p}\right]$ is a cocomplete 2 -category. So the conditions of Theorem 4 hold for Example 10, and so the inclusion

$$
\left[L, \text { Poset }^{o p}\right] \longrightarrow \operatorname{Lax}\left(L, \text { Poset }^{o p}\right)
$$

has a left adjoint. But $\left[L, \text { Poset }^{o p}\right]^{o p}$ is canonically isomorphic to $\left[L^{o p}\right.$, Poset $]$, and $\operatorname{Lax}\left(L, \text { Poset }^{o p}\right)^{o p}$ is canonically isomorphic to $\operatorname{Lax}\left(L^{o p}\right.$, Poset $)$, and in general, a functor $H: A \longrightarrow B$ has a right adjoint if and only if $H: A^{o p} \longrightarrow B^{o p}$ has a left adjoint. The combination of these facts yields the result.

With this result in hand, one can systematically work through Bonchi and Zanasi's paper, adapting their constructions for saturation and desaturation, without discarding the inherent laxness that logic programming, cf data refinement, possesses.

We have stated the results here for arbitrary lax transformations, but they apply equally to those that strictly respect injections, i.e., a subtle extension of the above argument shows that the inclusion

$$
\left[L^{o p}, \text { Poset }\right] \longrightarrow \operatorname{Lax}_{I n j}\left(L^{o p}, \text { Poset }\right)
$$

has a right adjoint, that right adjoint being a further variant of the right Kan extension that Bonchi and Zanasi used. The argument for lax naturality from the Introduction retains its force, so in Bonchi and Zanasi's sense, this does not yield compositionality of lax semantics, but it does further refine their analysis of saturation, eliminating more double counting.

\section{Conclusions}

For variable-free logic programs, in [20, we used the cofree comonad on $P_{f} P_{f}$ to model the coinductive trees generated by a logic program. The notion of coinductive tree had not been isolated at the time of writing of [20, or of [21, so we did not explicitly explain the relationship in [20, hence our doing so here, but the result was effectively in [20], just explained in somewhat different terms.

Using lax transformations, we extended the result in [21, albeit again not stating it explicitly but again explained explicitly here, to arbitrary logic programs, including existential programs a leading example being GC, as studied extensively by Sterling and Shapiro 31. The problem of existential clauses is well-known in the literature on theorem proving and within communities that use term-rewriting, TM-resolution or their variants. In TRS [33, existential variables are not allowed to appear in rewriting rules, and in type inference, the restriction to non-existential programs is common 11. In LP, the problem of handling existential variables when constructing proofs with TM-resolution marks the boundary between the theorem-proving and problem-solving aspects, as explained in Section 2 . 
The papers 2123] also contained a kind of category theoretic semantics for existential logic programs such as GC, but that semantics was limited, not modelling the coinductive trees generated by TM-resolution for such logic programs. Here, we have refined lax semantics, refining $\operatorname{Lax}\left(\mathcal{L}_{\Sigma}^{o p}\right.$, Poset $)$ to $\operatorname{Lax}{ }_{I n j}\left(\mathcal{L}_{\Sigma}^{o p}\right.$, Poset $)$, thus insisting upon strict naturality for injections, and refining the construction $P_{c} P_{f} A t$ to $P_{f f}(A t)$, thus allowing for additional variables in the tail of a clause in a logic program and not introducing countability, cf the modelling of local state in [29. This has allowed us to model coinductive trees for arbitrary logic programs.

We have further mildly refined Bonchi and Zanasi's saturation semantics for logic programming [6], showing how it may be seen to complement rather than to replace lax semantics.

\section{References}

1. G. Amato, J. Lipton, and R. McGrail, On the algebraic structure of declarative programming languages, Theor. Comput. Sci., 410(46):46264671, 2009.

2. J. Benabou, Introduction to bicategories. In Volume 47 of Lecture Notes in Mathematics, pages 1-77, Springer, 1967. Springer,

3. R. Blackwell, G.M. Kelly, and A.J. Power, Two-dimensional monad theory. J. Pure Appl. Algebra 59 (1989) 1-41.

4. F. Bonchi and U. Montanari, Reactive systems, (semi-)saturated semantics and coalgebras on presheaves. Theor. Comput. Sci., 410(41):40444066, 2009.

5. R. Bruni, U. Montanari, and F. Rossi, An interactive semantics of logic programming. TPLP, 1(6):647690, 2001.

6. F. Bonchi and F. Zanasi, Saturated semantics for coalgebraic logic programming. In CALCO, volume 8089 of Lecture Notes in Computer Science, pages 8094. Springer, 2013.

7. F. Bonchi and F. Zanasi. 2015. Bialgebraic Semantics for Logic Programming. CoRR abs/1502.06095 (2015).

8. M. Comini, G. Levi, and M. C. Meo, A theory of observables for logic programs. Inf. Comput., 169(1):2380, 2001.

9. P. Fu and E. Komendantskaya. 2015. A Type-Theoretic Approach to Resolution. In LOPSTR'15.

10. P. Fu and E. Komendantskaya and T. Schrijvers and A. Pond. 2015. Proof Relevant Corecursive Resolution. In FLOPS'16.

11. S. P. Jones, M. Jones, and E. Meijer. Type classes: An exploration of the design space. In In Haskell Workshop, 1997.

12. G. Gupta and V. Costa. Optimal implementation of and-or parallel prolog. In PARLE92, pages 7192, 1994.

13. He Jifeng and C.A.R. Hoare, Categorical semantics for programming languages, Proc MFPS 1989, in volume 442 of Lecture Notes in Computer Science, pages 402-417, Springer 1989.

14. He Jifeng and C.A.R. Hoare, Data refinement in a categorical setting, 1990. Technical Monograph PRG-90, Oxford University Computing Laboratory, Programming Research Group, Oxford, 1990.

15. P. Johann, E. Komendantskaya, and V. Komendantskiy. 2015. Structural Resolution for Logic Programming. In Technical Communications of ICLP'15. 
16. G.M. Kelly, Coherence theorems for lax algebras and for distributive laws. In Category seminar, volume 420 of Lecture Notes in Mathematics 420, pages 281-375, Spriniger, 1974.

17. G.M. Kelly, Basic concepts of enriched category theory, London Math. Soc. Lecture Notes Series 64, Cambridge University Press (1982).

18. Y. Kinoshita and A.J. Power, Lax naturality through enrichment, J. Pure Appl. Algebra 112 (1996) 53-72.

19. Y. Kinoshita and J. Power, A fibrational semantics for logic programs. In Proc. Int. Workshop on Extensions of Logic Programming, volume 1050 of LNAI, 1996.

20. E. Komendantskaya, G. McCusker, and J. Power, Coalgebraic semantics for parallel derivation strategies in logic programming. In AMAST2010, volume 6486 of Lecture Notes in Computer Science, pages 111-127, Springer, 2011.

21. E. Komendantskaya and J. Power, Coalgebraic semantics for derivations in logic programming. In CALCO 2011, volume 6859 of Lecture Notes in Computer Science, pages 268 - 282. Springer, 2011.

22. E. Komendantskaya and J. Power. Coalgebraic derivations in logic programming. In CSL, LIPIcs, pages 352-366. Schloss Dagstuhl, 2011.

23. E. Komendantskaya, J. Power and M. Schmidt, Coalgebraic Logic Programming:from Semantics to Implementation, J. Logic Computation (2014) doi: $10.1093 / \log c o m /$ exu026

24. E.Komendantskaya, M.Schmidt and J.Heras, Exploiting Parallelism in Coalgebraic Logic Programming. Electr. Notes Theor. Comput. Sci. 303: 121-148 (2014).

25. J. Lloyd. Foundations of Logic Programming. Springer, 2nd edition, 1987.

26. S. Mac Lane, Categories for the Working Mathematician, Graduate Texts in Mathematics, Springer, 1971.

27. A.J. Power, An algebraic formulation for data refinement, Proc MFPS 1989, in volume 442 of Lecture Notes in Computer Science, pages 390-401, Springer, 1989.

28. G.D. Plotkin and A.J. Power, Adequacy for Algebraic Effects. In FOSSACS 2001, volume 2030 of Lecture Notes in Computer Science, pages 1-24, Springer, 2001.

29. G.D. Plotkin and A.J. Power, Notions of computation determine monads. In Proc FOSSACS 2002, volume 2303 of Lecture Notes in Computer Science, pages 342-356, Springer, 2002.

30. L. Simon, A. Bansal, A. Mallya, and G. Gupta. Co-logic programming: Extending logic programming with coinduction. In Automata, Languages and Programming, pages 472-483. Springer, 2007.

31. L. Sterling and E. Shapiro. The art of Prolog. MIT Press, 1986.

32. Ross Street, The formal theory of monads, J. Pure Appl. Algebra 2 (1972) 149-168.

33. Terese. 2003. Term Rewriting Systems. Cambridge University Press.

34. J. Worrell, Terminal sequences for accessible endofunctors. In Proc CMCS 1999, volume 19 of Electronic Notes in Theoretical Computer Science (1999) 19:2438. 